\title{
Validation of a Piezoelectric Sensor Array-Based Device for Measurement of Carotid-Femoral Pulse Wave Velocity: The Philips Prototype
}

\author{
Shao-Kun $\mathrm{Xu}^{\mathrm{a}} \quad$ Xiang-Fei Hong ${ }^{\mathrm{b}} \quad$ Yi-Bang Cheng ${ }^{\mathrm{a}}$ Chang-Yuan Liu ${ }^{\mathrm{a}}$ \\ Yan Li ${ }^{a}$ Bin Yin ${ }^{b}$ Ji-Guang Wang ${ }^{a}$ \\ ${ }^{a}$ Center for Epidemiological Studies and Clinical Trials, Shanghai Key Laboratory of \\ Hypertension, The Shanghai Institute of Hypertension, Department of Hypertension, \\ Ruijin Hospital, Shanghai Jiaotong University School of Medicine, Shanghai, China;

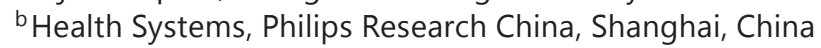

\section{Keywords}

Arterial stiffness · Carotid-femoral pulse wave velocity · Piezoelectric sensor array

\begin{abstract}
Background: Multiple piezoelectric pressure mechanotransducers topologized into an array might improve efficiency and accuracy in collecting arterial pressure waveforms for measurement of pulse wave velocity (PWV). Objective: In the present study, we validated a piezoelectric sensor array-based prototype (Philips) against the validated and clinically widely used Complior device (Alam Medical). Methods: We recruited 33 subjects with a wide distribution of PWV. For the validation, PWV was measured sequentially with the Complior device (four times) and the Philips prototype (three times). With the 99 paired PWV values, we investigated the agreement between the Philips prototype and the Complior device using Pearson correlation analysis and Bland-Altman plot. We also performed analysis on the determinants and reproducibility of PWV measured with both devices. Results: The correlation coefficient for PWV measured with the two devices was $0.92(p<0.0001)$. Compared with the Complior device, the Philips prototype slightly overestimated PWV by 0.24 ( \pm 2 standard deviations, \pm 1.91 ) $\mathrm{m} / \mathrm{s}$, especially when PWV was high. The correlation coefficient between the difference and the average of the Philips and Complior measurements was $0.21(p=0.035)$. Nonetheless, they had similar determinants. Age, mean arterial pressure, and sex altogether explained 81.6 and $83.9 \%$ of the variance of PWV values measured with the Philips prototype and Complior device, respectively. When the two extremes of the three PWV values measured with the Philips prototype and the Complior device were investigated, the coefficients of variation were 8.26 and $3.26 \%$, respectively. Conclusions: Compared with the Complior device, the Philips prototype had similar accuracy, determinants, and reproducibility in measuring PWV.


Xu et al.: Validation of a Device for Pulse Wave Velocity Measurement

\section{Introduction}

Current hypertension guidelines recommend arterial stiffness, measured by aortic pulse wave velocity (PWV), as an assessment of target organ damage [1] and a factor for risk stratification [2]. Indeed, aortic PWV may improve cardiovascular prediction in the general population [3] and in patients with various diseases, such as hypertension [4, 5], end-stage renal disease [6], etc. Aortic PWV is usually measured as carotid-femoral PWV by collecting the pulse waveforms on the carotid and femoral arteries and by measuring the distance between the two arterial sites. There is some discussion on measurement errors of the travel path length of the pulse waves and the influence on accuracy of carotid-femoral PWV measurement [7]. In fact, the collection of pulse waves may also impact the accuracy of measurement [8]. The piezoelectric pressure mechanotransducing technology (Complior; Alam Medical, France) allows recording of waveforms simultaneously on two superficial arterial sites and has relatively low cost compared to the Millar tonometry technique that records the carotid and femoral waveforms sequentially. However, a single sensor of the piezoelectric pressure mechanotransducing technology may not be sufficiently accurate in collecting pulse waveforms of carotid and femoral arteries and in turn also in measuring carotid-femoral PWV. One possible approach to improve efficiency and accuracy is to increase the number of sensors of the transducer.

The Philips prototype is designed with a piezoelectric sensor array in attempt to have an accurate, easy-to-use, and reliable low-cost solution for measurement of carotid-femoral PWV. It has to be validated for accuracy. To design a validation study, we referred to the European Society of Hypertension International Protocol for validation of blood pressure measuring devices in adults [9] regarding the selection of subjects in a wide range of carotidfemoral PWV and the repeated measurements in each study subject. We chose the validated and well-accepted Complior device as the standard [10]. We report in the present article the results of a validation study of the Philips prototype against the Complior device in subjects with a wide range of carotid-femoral PWV.

\section{Methods}

\section{Study Population}

Our study subjects were recruited in the specialized outpatient clinic of hypertension, Ruijin Hospital, Shanghai Jiaotong University School of Medicine, Shanghai, China. We screened consecutive subjects aged 25-75 years for carotid-femoral PWV, measured by the Complior device, and enrolled those with a PWV in the range from 5 to $15 \mathrm{~m} / \mathrm{s}$. We excluded patients with atrial fibrillation. For practical reasons, we also excluded patients with known secondary hypertension, peripheral vascular disease, cerebrovascular disease, valvular heart disease, cardiac arrhythmia, congestive heart failure, or any other life-threatening diseases.

The Philips Prototype

The Philips prototype is composed of two PWV measurement probes and one ECG probe (with three electrodes). The system estimates the pulse transit time by a foot-to-foot method, operates the applanation tonometry technology, and simultaneously collects carotid and femoral waveforms with two piezoelectric probes. The carotid and femoral probes consist of 4 and 5 piezoelectric sensors, respectively. Each sensor consists of an integrated piezoelectric membrane. The probe chooses the best signal detected by one of the multiple sensors. An acquisition module transmits the data to a MatLab program for data storage and analysis.

The Validation Protocol

We chose the widely used Complior device as the standard and measured PWV sequentially four times with the Complior device (PWV measurements 1,3,5, and 7) and three times with the Philips prototype (PWV measurements 2, 4, and 6). After the enrolled subjects had rested in the supine position for at least $5 \mathrm{~min}$, an experienced technician measured his or her blood pressure three times consecutively using a validated automated upper arm blood pressure monitor (HEM-7011; Omron, Kyoto, Japan). A technician 
Xu et al.: Validation of a Device for Pulse Wave Velocity Measurement

Table 1. Characteristics of the study participants

\begin{tabular}{lccc}
\hline \multirow{2}{*}{ Characteristic } & \multicolumn{3}{c}{ All subjects (17 men and 16 women) } \\
\cline { 2 - 4 } & mean \pm SD & minimum & maximum \\
\hline Age, years & $46.6 \pm 12.8$ & 25 & 60 \\
Body height, cm & $166.3 \pm 8.2$ & 150 & 183 \\
Body weight, kg & $63.9 \pm 10.8$ & 45 & 91 \\
Body mass index & $23.0 \pm 2.3$ & 18.0 & 27.4 \\
Systolic blood pressure, mm Hg & $132.3 \pm 20.9$ & 99 & 180 \\
Diastolic blood pressure, mm Hg & $73.0 \pm 10.1$ & 60 & 97 \\
Mean arterial pressure, mm Hg & $92.8 \pm 13.0$ & 74.7 & 124.7 \\
Heart rate, beats/min & $71.6 \pm 7.7$ & 59 & 94 \\
Carotid-femoral distance, cm & $64.0 \pm 4.0$ & 57 & 70 \\
Carotid-femoral pulse wave velocity, m/s & & & 14.9 \\
\multicolumn{1}{c}{ Complior device ( mean of four readings) } & $10.63 \pm 2.20$ & 5.9 & 18.48 \\
\hline
\end{tabular}

SD, standard deviation.

measured the distance from the sternal notch to the femoral site and the distance from the sternal notch to the carotid site. The length of the travel path was the sum of the two distances and was used for the calculation of PWV for both devices. Carotid and femoral pulse waveforms were then collected alternatively with the Complior device and the Philips prototype, and the time delay between carotid and femoral pulse waves was computed. PWV was calculated by dividing the length of the travel path by the time delay of the pulse waves. Mean arterial pressure was calculated as the sum of one-third of systolic blood pressure and twothirds of diastolic blood pressure.

\section{Statistical Analysis}

We used SAS software version 9.2 (SAS Institute, Cary, NC, USA) for data management and statistical analysis. The difference in PWVs between the Complior device and the Philips prototype was calculated by subtracting the PWV values measured with the Philips prototype from the preceding and subsequent PWV values measured with the Complior device. The paired values with the smaller difference were selected for analysis. For the 99 pairs, agreement was analyzed with the Bland-Altman approach by plotting the difference between the Complior device and the Philips prototype against the average of the PWV values obtained with the two devices. Pearson correlation analysis was also performed by correlating the 99 values obtained by the Philips prototype with the 99 values obtained by the Complior device.

In further analysis, we averaged the three measurements per study subject for the Complior device and the Philips prototype, respectively. In these 33 subjects, we performed multiple stepwise regression analysis to study the determinants of PWV obtained with the two devices. The $p$ value for covariables to enter and stay in the model was set at 0.10 . We compared the size of the correlation coefficients after Fisher $z$ transformation [11]. We also performed reproducibility analysis of the two extreme values of the three PWV measurements per device in the 33 subjects. We computed coefficients of variation as the ratio of the standard deviation to the mean of the difference of the PWV values.

\section{Results}

\section{Characteristics of the Study Participants}

The 33 study participants included 17 (51.5\%) men, 10 (30.3\%) treated hypertensive patients, and $2(6.1 \%)$ diabetic patients. They had a mean age of $46.6 \pm 12.8$ years, a mean body mass index of $23.0 \pm 2.3$, a mean systolic/diastolic blood pressure of $132.3 / 73.0 \mathrm{~mm} \mathrm{Hg}$, and a mean heart rate of $71.6 \pm 7.7$ beats/min (Table 1 ). The proportion of current smoking and alcohol intake was $36.4 \%(n=12)$ and $21.2 \%(n=7)$, respectively. 
Fig. 1. Correlation between pulse wave velocity (PWV) measured with the Philips prototype and the Complior device. The regression line is shown. The regression equation is given with correlation coefficient and $p$ value.
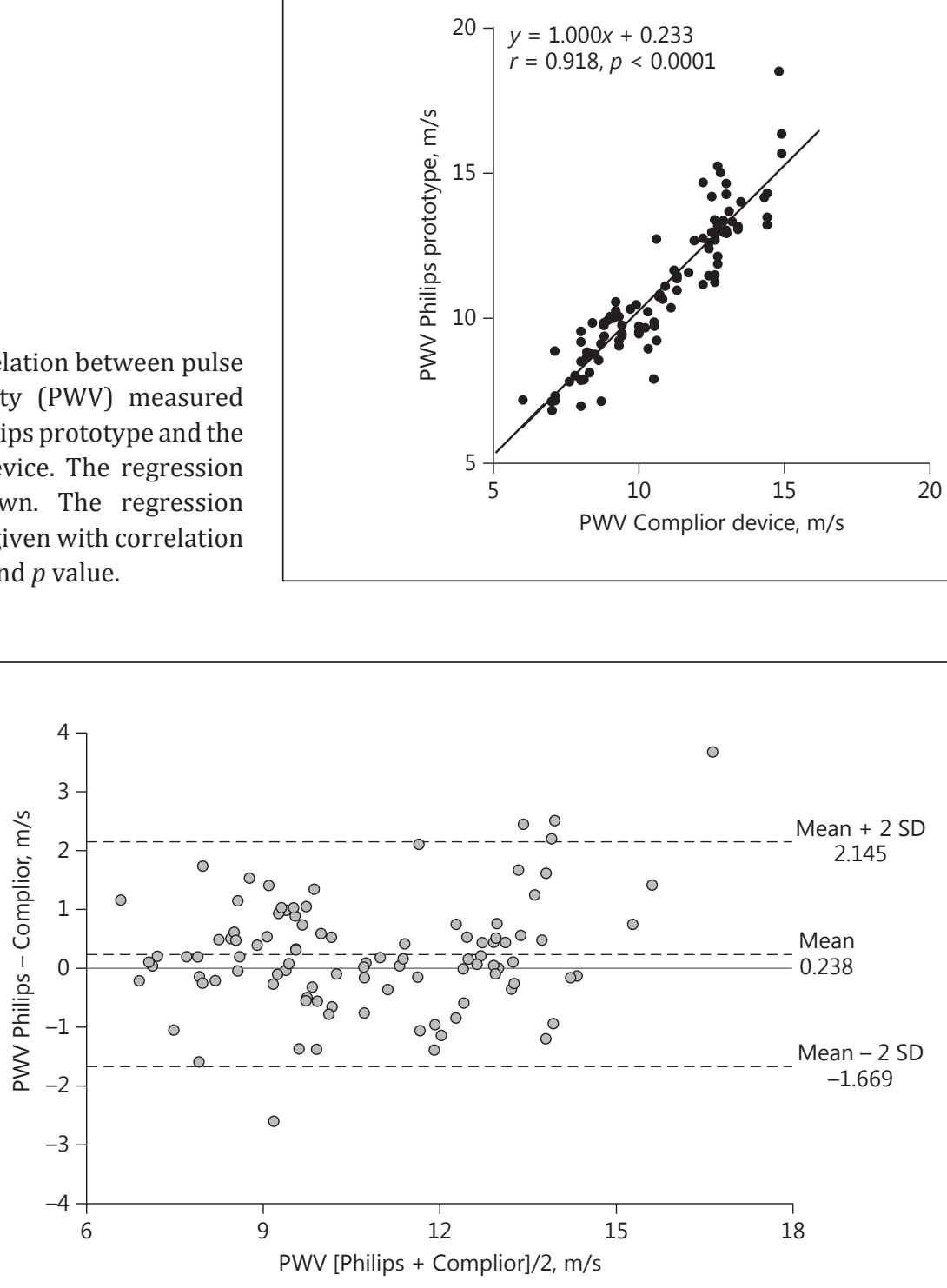

Fig. 2. Bland-Altman analysis of the difference between pulse wave velocity (PWV) values obtained by the two devices against the average of the two PWV values. The lines of the mean difference and the mean \pm 2 standard deviations (SD) are shown.

The mean pulse wave travel distance was $64.0 \pm 4.0 \mathrm{~cm}$ (range $57-70 \mathrm{~cm}$ ). The first PWV measurement obtained with the Complior device averaged $10.6 \mathrm{~m} / \mathrm{s}$ (range $5.9-14.9 \mathrm{~m} / \mathrm{s}$ ) and was $<10 \mathrm{~m} / \mathrm{s}$ in 10 subjects, $10-11 \mathrm{~m} / \mathrm{s}$ in 8 subjects, and $>11 \mathrm{~m} / \mathrm{s}$ in 15 subjects.

\section{Agreement between the Philips Prototype and the Complior Device}

Among the 99 pairs, there was significant correlation between PWV values obtained with the Philips prototype and the Complior device, with a correlation coefficient of $0.92(p<$ 0.0001) (Fig. 1). The mean difference (95\% confidence interval) in PWV values between the two devices was 0.24 ( -1.67 to 2.14 ) m/s (Fig. 2). The difference was significantly associated 
Xu et al.: Validation of a Device for Pulse Wave Velocity Measurement

Table 2. Regression analysis on the determinants of pulse wave velocity measured with the Philips prototype and the Complior device

\begin{tabular}{|c|c|c|c|c|c|c|c|}
\hline \multirow[t]{2}{*}{ Variable } & \multicolumn{3}{|c|}{ Philips prototype } & \multicolumn{3}{|c|}{ Complior device } & \multirow[t]{2}{*}{$p_{\mathrm{r}}$} \\
\hline & partial $r$ & $\beta \pm S E, m / s$ & $p$ & partial $r$ & $\beta \pm S E, m / s$ & $p$ & \\
\hline Age,+10 years & 0.44 & $1.23 \pm 0.19$ & $<0.0001$ & 0.43 & $0.91 \pm 0.15$ & $<0.0001$ & 0.94 \\
\hline $\mathrm{MAP},+10 \mathrm{~mm} \mathrm{Hg}$ & 0.76 & $0.70 \pm 0.18$ & 0.0004 & 0.80 & $0.81 \pm 0.16$ & $<0.0001$ & 0.53 \\
\hline Male sex & 0.21 & $1.36 \pm 0.42$ & 0.003 & 0.13 & $0.65 \pm 0.37$ & 0.091 & 0.59 \\
\hline Body mass index & -0.15 & $-0.21 \pm 0.10$ & 0.05 & - & - & - & - \\
\hline
\end{tabular}

In multiple stepwise regression analysis we considered sex, age, mean arterial pressure, heart rate, body mass index, smoking, and drinking. We reported partial $r$, regression coefficient $(\beta) \pm$ standard error (SE), and $p$ value for variables entered and staying in the model. $p_{\mathrm{r}}$ is for the difference in correlation coefficients between the two devices. MAP, mean arterial pressure.

with the mean of PWV values measured with the two devices, with a correlation coefficient of 0.21 ( $p=0.035$ ), indicating under- and overestimation of PWV with the Philips prototype at low and high levels of PWV, respectively (Fig. 2).

Determinants of PWV Obtained with the Philips Prototype and the Complior Device

In multiple stepwise regression analysis, we considered age, sex, body mass index, mean arterial pressure, heart rate, current smoking, and alcohol intake as determinants of PWV.We found that PWV was associated with age, sex, and mean arterial pressure for both devices $(p \leq 0.09)$ and with body mass index for the Philips prototype $(p=0.05)$. These variables altogether explained 81.6 and $83.9 \%$ of the variance for the Philips prototype and the Complior device, respectively (Table 2). The relationship between PWV and its major determinants, such as age and mean arterial pressure, was indistinguishable between the two devices (Fig. 3). We also investigated the difference in the magnitude of correlation coefficients of the major determinants of PWV between the two devices. None of the differences in the magnitude of correlation coefficients between the two devices reached statistical significance $(p \geq 0.53)$ (Table 2).

\section{Reproducibility of PWV Values Obtained with the Philips Prototype and the Complior}

Device

Reproducibility was investigated with the two extreme values of the three PWV measurements per device. The coefficients of variation were 8.26 and $3.26 \%$ for the Philips prototype and the Complior device, respectively.

\section{Discussion}

Our study showed that, compared with the Complior device, the Philips prototype had similar accuracy, determinants, and reproducibility in PWV measurement. Indeed, the correlation coefficient was as high as 0.92 , and the mean difference was as small as $0.24 \mathrm{~m} / \mathrm{s}$. Measurements with both devices were highly dependent on age, mean arterial pressure, and sex, and were reproducible.

According to the ARTERY Society guidelines on validation of devices for the noninvasive measurement of arterial PWV, the accuracy of measurement with a test device is charac- 


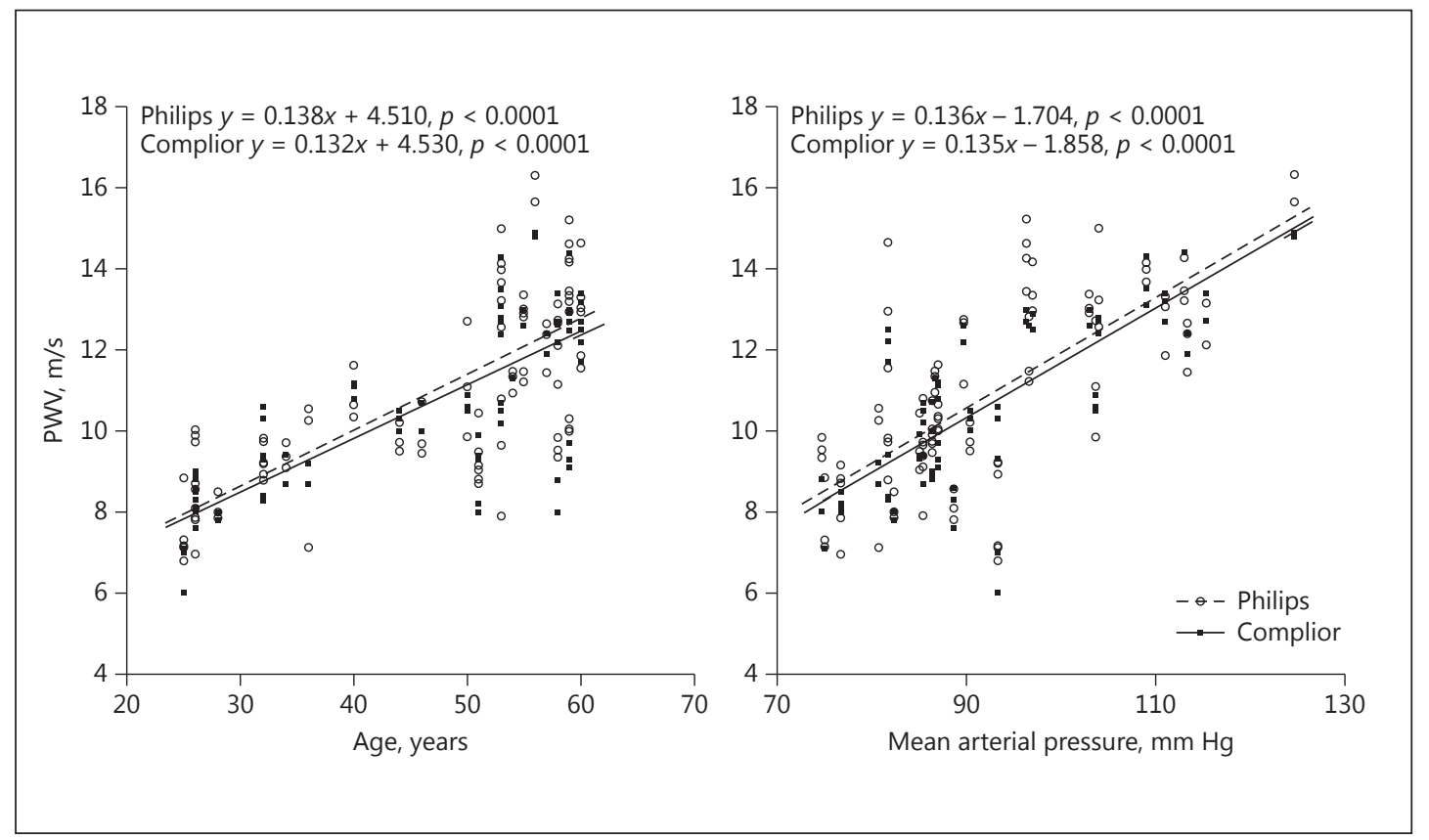

Fig. 3. Pulse wave velocity (PWV) measured with the Philips prototype (open circles) and the Complior device (full squares) in relation to age (left panel) and mean arterial pressure (right panel). Regression lines are shown separately for the Philips prototype (dashed line) and the Complior device (full line). The regression equation is also given with the $p$ value of the regression coefficient for the two devices separately.

terized as "acceptable" when the mean difference is $<1.0 \mathrm{~m} / \mathrm{s}$ and the standard deviation of the difference between the test and standard device is $<1.5 \mathrm{~m} / \mathrm{s}$ [12]. The Philips prototype should be considered more than "acceptable" (mean difference $0.24 \mathrm{~m} / \mathrm{s}$, standard deviation $0.95 \mathrm{~m} / \mathrm{s}$ ). Nonetheless, our study was not conducted in complete accordance with this guideline document. It included 33 subjects, instead of the 90 or more subjects recommended by the guideline. However, we performed repeated measurements for both the test and the standard device, and eventually 99 paired measurements were included in the analysis of accuracy. In addition, our study subjects had a wide distribution of PWV measured with the standard device. Finally, the PWV measurements in our study were performed by a single instead of two technicians. One experienced technician may have systemic errors in measurements, for instance in measuring the distance between the two arterial sites. However, such systemic error, if any, should be similar for both devices.

The analysis on the major determinants of PWV measured with the two devices also supports the consistency in the measurement of PWV between the two devices. The determinants of PWV values measured with the two devices are almost identical regarding the size of the partial correlation coefficients and the adjusted regression coefficients. Nonetheless, body mass index was significantly associated with PWV measured with the Philips prototype, but not with the Complior device. In fact, our finding is in keeping with the result of a previous validation study on the Complior device in which body mass index was not associated with PWV [13]. Given the weak correlation between body mass index and PWV measured with the Philips prototype, a chance finding is also possible. Moreover, the reproducibility analysis is also supportive. Both devices had a coefficient of variation $<10 \%$. It is noteworthy that this reproducibility assessment was performed with two extremes of the three measurements included in the analysis. 
In conclusion, the Philips prototype is in close agreement with the Complior device in the measurement of carotid-femoral PWV. If developed into a commercial product, it may be an easy-to-use and low-cost tool for measurement of carotid-femoral PWV. Such a device may offer the possibility to measure PWV in low-resource settings such as primary care practices. Our study was conducted in the Chinese population. Whether the results of our validation study can be extrapolated to other populations or ethnicities remains under investigation. Nonetheless, taken the fact that the Complior device has been widely used and accepted in many different populations and ethnicities [13-15], our results may also be applicable to other ethnicities.

\section{Acknowledgments}

The authors gratefully acknowledge the voluntary contribution of the study subjects and the expert help of Jun-Wei Li (The Shanghai Institute of Hypertension, Shanghai, China) and Sandy Shen (Philips Research China, Shanghai, China).

\section{Statement of Ethics}

The study protocol was approved by the ethics committee of Ruijin Hospital, Shanghai Jiaotong University School of Medicine. All subjects gave written informed consent.

\section{Disclosure Statement}

Dr. J.-G. Wang reports receiving lecture and consulting fees from Daiichi-Sankyo, Novartis, Omron, Pfizer, Sanofi, and Servier. There are no conflicts of interest for the other authors.

\section{Funding Sources}

Philips Research China (Shanghai, China) provided funding for this validation study.

\section{References}

1 Mancia G, Fagard R, Narkiewicz K, Redon J, Zanchetti A, Bohm M, Christiaens T, Cifkova R, De Backer G, Dominiczak A, Galderisi M, Grobbee DE, Jaarsma T, Kirchhof P, Kjeldsen SE, Laurent S, Manolis AJ, Nilsson PM, Ruilope LM, Schmieder RE, Sirnes PA, Sleight P, Viigimaa M, Waeber B, Zannad F: 2013 ESH/ESC guidelines for the management of arterial hypertension: the task force for the management of arterial hypertension of the European Society of Hypertension (ESH) and of the European Society of Cardiology (ESC). J Hypertens 2013;31:1281-1357.

2 Liu LS: 2010 Chinese guidelines for the management of hypertension. Chin J Cardiol 2011;39:579-615.

-3 Willum-Hansen T, Staessen JA, Torp-Pedersen C, Rasmussen S, Thijs L, Ibsen H, Jeppesen J: Prognostic value of aortic pulse wave velocity as index of arterial stiffness in the general population. Circulation 2006;113: 664-670.

4 Sheng CS, Li Y, Li LH, Huang QF, Zeng WF, Kang YY, Zhang L, Liu M, Wei FF, Li GL, Song J, Wang S, Wang JG: Brachial-ankle pulse wave velocity as a predictor of mortality in elderly Chinese. Hypertension 2014;64: 1124-1130.

5 Boutouyrie P, Tropeano AI, Asmar R, Gautier I, Benetos A, Lacolley P, Laurent S: Aortic stiffness is an independent predictor of primary coronary events in hypertensive patients: a longitudinal study. Hypertension 2002;39:10-15.

6 London GM, Blacher J, Pannier B, Guérin AP, Marchais SJ, Safar ME: Arterial wave reflections and survival in end-stage renal failure. Hypertension 2001;38:434-438.

7 Weber T, Hametner B, Wassertheurer S: Travel distance estimation for carotid femoral pulse wave velocity: is the gold standard a real one? J Hypertens 2011;29:2491-2493. 
8 Davies JM, Bailey MA, Griffin KJ, Scott DJ: Pulse wave velocity and the non-invasive methods used to assess it: Complior, SphygmoCor, Arteriograph and Vicorder. Vascular 2012;20:342-349.

-9 O’Brien E, Atkins N, Stergiou G, Karpettas N, Parati G, Asmar R, Imai Y, Wang J, Mengden T, Shennan A; Working Group on Blood Pressure Monitoring of the European Society of Hypertension: European Society of Hypertension International Protocol revision 2010 for the validation of blood pressure measuring devices in adults. Blood Press Monit 2010;15:23-38.

10 Stea F, Bozec E, Millasseau S, Khettab H, Boutouyrie P, Laurent S: Comparison of the Complior Analyse device with Sphygmocor and Complior SP for pulse wave velocity and central pressure assessment. J Hypertens 2014;32:873-880.

11 Bland JM, Altman DG: Measurement error and correlation coefficients. BMJ 1996;313:41-42.

12 Wilkinson IB, McEniery CM, Schillaci G, Boutouyrie P, Segers P, Donald A, Chowienczyk PJ; on behalf of the ARTERY Society: ARTERY Society guidelines for validation of non-invasive haemodynamic measurement devices: Part 1, arterial pulse wave velocity. Artery Res 2010;4:34-40.

13 Asmar R, Benetos A, Topouchian J, Laurent P, Pannier B, Brisac AM, Target R, Levy BI: Assessment of arterial distensibility by automatic pulse wave velocity measurement. Validation and clinical application studies. Hypertension 1995;26:485-490.

14 Blacher J, Asmar R, Djane S, London GM, Safar ME: Aortic pulse wave velocity as a marker of cardiovascular risk in hypertensive patients. Hypertension 1999;33:1111-1117.

15 Asmar R, Topouchian J, Pannier B, Benetos A, Safar M: Pulse wave velocity as endpoint in large-scale intervention trial. The Complior Study. Scientific, Quality Control, Coordination and Investigation Committees of the Complior Study. J Hypertens 2001;19:813-818. 